\title{
Comparing the effects of an acute bout of physical exercise with an acute bout of interactive mental and physical exercise on electrophysiology and executive functioning in younger and older adults
}

\author{
Julia Dimitrova ${ }^{1,4}$ (1) Michael Hogan $^{2} \cdot$ Patrick Khader $^{1} \cdot$ Denis O'Hora $^{2}$ • \\ Liam Kilmartin ${ }^{2} \cdot$ Jane C. Walsh $^{2} \cdot$ Richard Roche $^{5} \cdot$ Cay Anderson-Hanley ${ }^{3}$
}

Received: 20 September 2016/ Accepted: 9 November 2016/Published online: 19 November 2016

(C) Springer International Publishing Switzerland 2016

\begin{abstract}
Background Physical exercise has been shown to improve cognitive and neural functioning in older adults.

Aims and methods The current study compared the effects of an acute bout of physical exercise with a bout of interactive mental and physical exercise (i.e., "exergaming") on executive (Stroop) task performance and event-related potential (ERP) amplitudes in younger and older adults.

Results Results revealed enhanced executive task performance in younger and older adults after exercise, with no differences in performance between exercise conditions. Stroop (RT) performance in older adults improved more than in younger adults from pre- to post-exercise. A significant increase in EEG amplitude from pre- to post-exercise was found at the $\mathrm{Cz}$ site from 320 to $700 \mathrm{~ms}$ poststimulus for both younger and older adults, with older adults demonstrating a larger Stroop interference effect. While younger adults exhibited overall greater EEG amplitudes than older adults, they showed no differences between congruent and incongruent trials (i.e., minimal interference). Compared to peers with higher BMI (body mass index), older adults with lower BMI showed a greater reduction in Stroop interference effects from pre- to postexercise.
\end{abstract}

\footnotetext{
Julia Dimitrova

julia.dimitrova@camh.ca

Ludwig-Maximillians-University Munich, Munich, Germany

2 National University Ireland, Galway, Ireland

3 Union College, Schenectady, NY, USA

4 Centre for Addiction and Mental Health, 1001 Queen Street West, Unit 4-1, Toronto, ON M6J1H4, Canada

5 National University of Ireland, Maynooth, Ireland
}

Discussion and conclusions The beneficial effects of an acute bout of physical exercise on cognitive and neural functioning in younger and older adults were confirmed, with no difference between standard exercise and exergaming. Findings suggest that BMI, sometimes used as a proxy for fitness level, may modulate benefits that older adults derive from an acute bout of exercise. Findings have implications for future research that seeks to investigate unique effects of exergaming when compared to standard physical exercise.

Keywords Aging $\cdot$ Stroop task $\cdot$ Interference $\cdot$ Mental and aerobic exercise

\section{Introduction}

Age-related cognitive decline has been well documented, with numerous studies reporting age-related declines in executive functioning and related abilities [1]. Research also highlights the cognitive benefits of physical exercise for older adults [2, 3]. Although both acute bouts of exercise and physical fitness established over a longer period of time have been shown to enhance cognitive functioning, the effects of physical fitness appear larger and more robust $[4,5]$. Nevertheless, a recent meta-analysis revealed that cognitive performance during, immediately after and after a delay of up to $20 \mathrm{~min}$ of an acute bout of exercise was enhanced and that these effects are larger for older adults when compared with younger adults [6].

Research consistently reports age-related deficits in the ability to inhibit distractions [7-9]. The current study employed electroencephalography (EEG) as a means to explore the neural mechanisms associated with inhibitory control and how these mechanisms may be differentially 
affected by standard physical exercise versus interactive mental and physical exercise (i.e., exergaming).

Anderson-Hanley et al. [10] conducted a long-term exercise intervention where older adults engaged in a program of exergaming. The results of this study demonstrated greater benefits of exergaming on measures of executive functioning compared with older adults enrolled in a program of standard physical exercise. While this and other studies [11] suggest the potential for unique benefits of exergaming, the neural underpinnings linking exergaming to improvements in cognitive functioning have yet to be examined. Furthermore, there have been no studies examining differences between younger and older adults and the effects of acute bouts of exergaming versus standard physical exercise. Therefore, the current study examined the effects of a single bout of physical exercise compared with a single bout exergaming on executive task performance and event-related potential (ERP) amplitudes in younger and older adults. Participants exercised on a recumbent stationary bicycle equipped with a virtual reality screen (the "cybercycle," Fig. 1). Half of the participants were randomly assigned to exercise on a virtual reality track, simply cycling around a loop with little to no cognitive stimulation; the other half were randomly assigned to a cycling condition requiring them to strategize and employ cognitive resources so as to score the most points possible in a dragon chase game.

Based on previous research, we expected to see improved cognitive performance in both younger and older adult groups in response to both exercise conditions. Furthermore, consistent with the results of a recent meta-analysis [6], we hypothesized that older adults would derive greater cognitive benefits from exercise compared with younger adults. Finally, based on available research $[10,11]$, we predicted that participants in the exergaming condition would receive an added benefit compared to those in the standard track condition. The executive functioning task selected for inclusion in the current study was the Stroop task, which has a well-identified electrophysiological signature. In the electrophysiological data, we predicted an attenuated Stroop interference effect post-exercise (i.e., increased positivity in mean amplitudes for congruent trials relative to incongruent trials) due to more efficient processing of incongruent stimuli $[12,13]$. We expected that, relative to younger adults, older adults would demonstrate a greater attenuation of the interference effect as a result of exercise. Based on previous work highlighting interactive effects of physical fitness and acute bouts of exercise on electrophysiological and cognitive outcomes [14], we also explored the moderating effects of body mass index (BMI), often used as a proxy for physical fitness, on cognitive and electrophysiological outcomes in younger and older adults.

\section{Methods}

\section{Participants}

Sixty older and younger adults participated in the study. Older adults were recruited from active retirement associations in the community and through television and newspaper announcements. Younger adults were recruited from the university population and local community. See Table 1 for participant demographics. Fifty-six participants were right-handed (four left-handed) and all had normal or corrected-to-normal vision. Participants were screened for a history of neurological or psychiatric disorders, as well as various medical conditions and medication intake. One older adult and one younger adult were excluded due to
Fig. 1 Model, Expresso S3R, "cybercycle" and its virtual reality features. Reprinted from Interactive Fitness Holdings, LLC (IFH) under a CC BY license, with permission from Interactive Fitness Holdings, LLC, original copyright 2011. a The Expresso S3R is a recumbent, stationary bicycle with a virtual reality display. b Track condition (physical exercise only condition). c Exergaming condition (combined mental and physical exercise condition): the dragon chase game

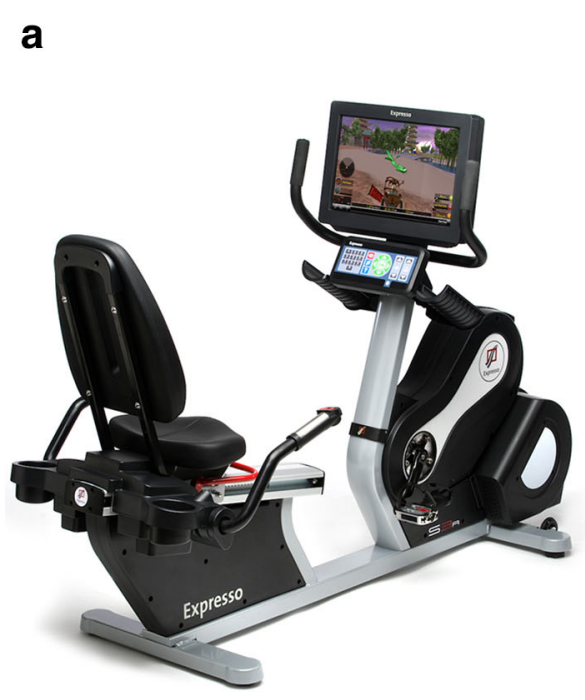

\section{b}

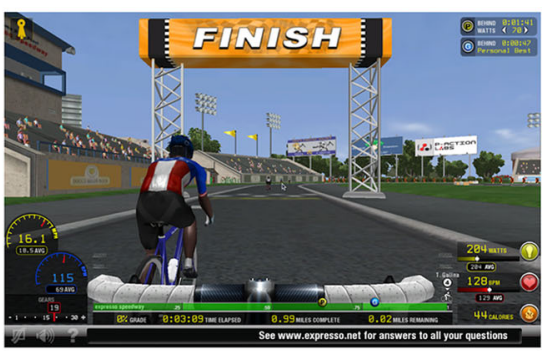

c

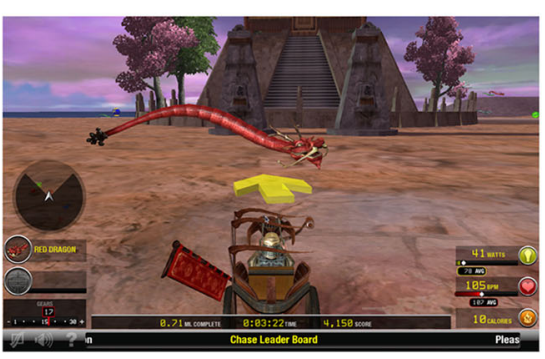


Table 1 Demographic characteristics of the sample

\begin{tabular}{llll}
\hline & Younger adults & Older adults & $p$ \\
& $N=29$ & $N=29$ \\
$M(\mathrm{SD})$ & \\
\hline Gender & $12 \mathrm{f}$ & $14 \mathrm{f}$ & n.s. \\
Age & $23.2(2.7)$ & $70.7(5.4)$ & $p<0.001$ \\
Education & $16.96(2.0)$ & $14.43(2.36)$ & $p<0.001$ \\
BMI & $24.7(5.1)$ & $28.1(3.96)$ & $p=0.008$ \\
Height (cm) & $174.7(6.2)$ & $166.3(9.2)$ & $p<0.001$ \\
Weight (kg) & $79.1(3.7)$ & $78.1(14.3)$ & n.s. \\
Digit span (backward) pre & $7.2(1.4)$ & $7.13(2.2)$ & n.s. \\
Digit span (backward) post & $7.6(1.4)$ & $6.8(2.4)$ & n.s. \\
Experience with computers (1-5) & $4.8(0.6)$ & $3.2(1.3)$ & $p<0.001$ \\
Experience with stationary exercise bike (1-5) & $2.9(0.7)$ & $2.3(1.2)$ & $p=0.008$ \\
Motivation to participate (0-100\%) & $80.6(18.2)$ & $85(20.6)$ & n.s. \\
\hline
\end{tabular}

Demographic information and participant characteristics, including means (SDs) of participant performance on the digit span task, as well as ratings of how motivated they were to participate and previous experience with computers and stationary exercise bicycles difficulties with the cognitive task and due to equipment failure, respectively. None of the participants were restricted by their physician from engaging in physical exercise. The study was approved by the National University of Ireland, Galway Research Ethics Committee and all participants provided written informed consent.

\section{Study design}

Participants first completed a paper and pencil forward and backward digit span. A computerized version of the Stroop task was then completed with simultaneous EEG recording. Participants were then randomly assigned to either the track condition or the game condition. In both conditions, participants rode the cybercycle for $20 \mathrm{~min}$. Participants were given a target heart rate to maintain during exercise using the heart rate reserve (HRR) method and were given regular feedback to maintain a relative HRR of $60 \%$. This resulted in a workout of moderate intensity relative to each person's resting HR and age [15]. There were no significant differences between the two exercise conditions in HRR.

Participants wore the EEG electrode cap throughout their exercise session. Participants were led to the EEG recording room after their exercise session and completed the Stroop task and digit span task, within 20 min of the intervention.

\section{Stroop task}

Participants completed a version of the Stroop task adapted for EEG from Kerns et al. [16]. In each trial, a central fixation cross was first presented for $500 \mathrm{~ms}$. Participants were then required to identify the color of words presented in one of three colors (red, blue or green) presented in the center of the screen, by pressing one of three color-coded keys on the computer keyboard (Fig. 2). The stimulus remained on the screen until response. The inter-trial interval was $1000 \mathrm{~ms}$.

A total of 198 color words were presented on screen and split into two blocks: congruent (color and word match) $=138$ trials and incongruent (color and word mismatch) $=60$ trials.

\section{Body mass index}

Participants' height and weight were measured at the time of testing so as to calculate BMI. Participants also completed an exercise history questionnaire at the beginning of each session, which included questions about their daily physical activity, including length of duration of each exercise session; type of exercise (e.g., strength and resistance or flexibility training) they engage in, the intensity at which they exercise; and their motivations for exercising. We found that there was a significant negative association $\left(r_{s}=-0.34, p<0.01\right)$ between participants' self-reported level of daily physical activity and their BMI.

\section{Electrophysiological recording and analysis}

Brain electrical activity was continuously recorded using a cap with 32 electrodes (Brain Products GmbH, Gilching, Germany). The electrode array conformed to the International 10-20 system. Vertical eye movements were recorded using two electrodes placed above and below the right eye, while electrodes at the outer canthus of each eye recorded horizontal eye movements. Data were sampled at a rate of $1000 \mathrm{~Hz}$, the band-pass filter applied ranged from 0 to $280 \mathrm{~Hz}$, and impedance was kept below $10 \mathrm{k} \Omega$. An online average reference was used and kept for off-line 
Fig. 2 Schematic of the computerized version of the Stroop task, adapted from Kerns et al. [16]

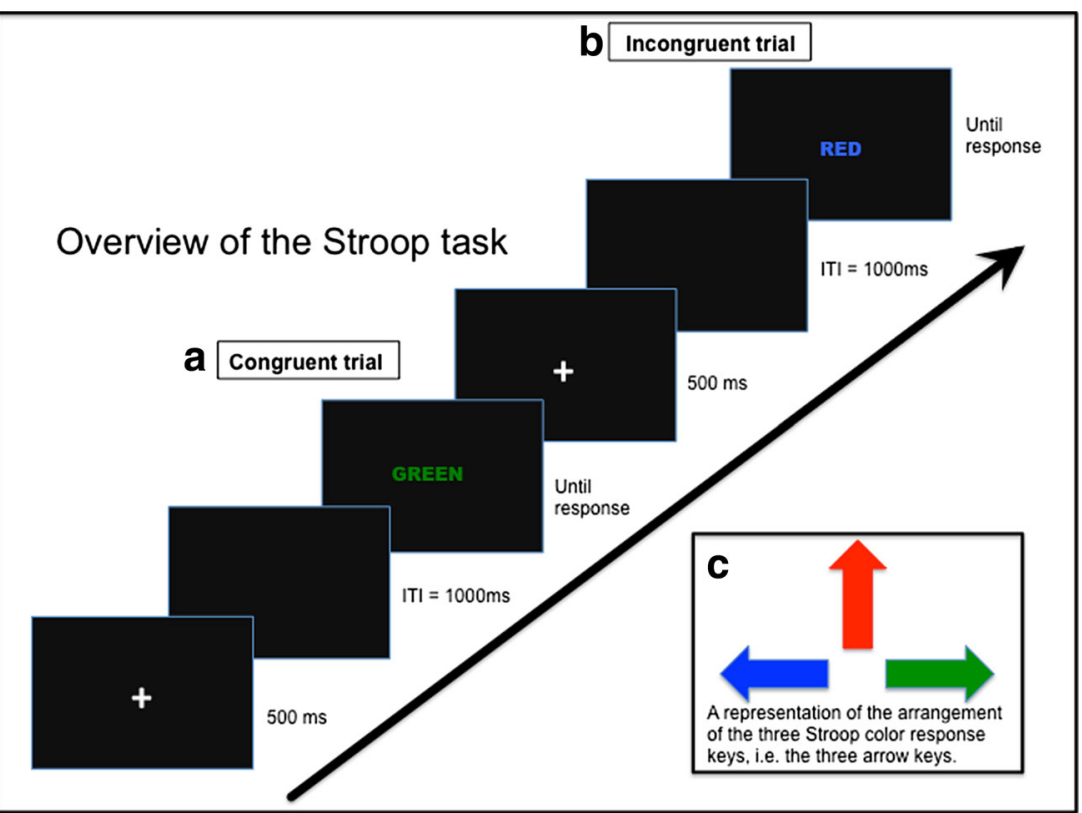

preprocessing and analysis. Epochs with eye movements and artifacts were rejected or corrected off-line.

Data were preprocessed using Brain Vision Analyzer. Noisy channels were removed from the data set and interpolated by means of spline interpolation. Data were highpass-filtered at $0.05 \mathrm{~Hz}$ and low-pass-filtered at $50 \mathrm{~Hz}$. Eye blink and movement artifacts were corrected for using the ocular correction algorithm by Gratton and Coles [17]. Further artifact rejection was applied, excluding epochs outside of the range $\pm 100 \mu \mathrm{V}$, allowing a maximal voltage step of $60 \mu \mathrm{V} / \mathrm{ms}$ and a maximal allowed difference of values in 200-ms intervals of $200 \mu \mathrm{V} / \mathrm{ms}$. Correct and artifactfree trials were segmented using stimulus-locked epochs: $100 \mathrm{~ms}$ before onset to $1000 \mathrm{~ms}$ post-stimulus onset. All trials were baseline corrected using the pre-stimulus interval as the baseline interval and then averaged.

\section{Statistical analysis}

Linear mixed effect models were employed to model behavioral reaction time (RT) and event-related potential (ERP) amplitudes during the Stroop task to examine the effects of Time (Pre and Post), Age (Young and Old), Condition (Track and Game) and Congruency (Congruent Stroop trials and Incongruent Stroop trials).

\section{Results}

Behavioral and electrophysiological performance on the Stroop task is presented in Table 2. We did not find any significant change in error rates from pre- to post-exercise across exercise conditions and groups.
Table 2 Behavioral and electrophysiological performance, including means (SDs) on the Stroop task

\begin{tabular}{llll}
\hline & $\begin{array}{l}\text { Young adults } \\
M(\mathrm{SD})\end{array}$ & $\begin{array}{l}\text { Older adults } \\
M(\mathrm{SD})\end{array}$ & $\begin{array}{l}\text { All } \\
\text { (SD) }\end{array}$ \\
\hline Behavioral & & & \\
Congruent (pre) & $637(117)$ & $1106(375)$ & \\
Congruent (post) & $549(81)$ & $862(256)$ & \\
Incongruent (pre) & $751(154)$ & $1462(511)$ & \\
Incongruent (post) & $654(133)$ & $1182(436)$ & \\
Errors (pre) & $8(6)$ & $8(11)$ & \\
Errors (post) & $7(5)$ & $3(3)$ & \\
Stroop effect (pre) & $114(76)$ & $355(222)$ & - \\
Stroop effect (post) & $105(66)$ & $321(231)$ & - \\
Stroop effect (overall) & $135(218)$ & $312(222)$ & - \\
Overall RT (pre) & $694(132)$ & $1284(434)$ & $991(299)$ \\
Overall RT (post) & $601(105)$ & $1022(339)$ & $813(228)$ \\
RT reduction (pre-post) & $93(79)$ & $262(202)$ & - \\
Electrophysiological & & & - \\
Congruent & $4(2)$ & $3(2)$ & - \\
Incongruent & $4(2)$ & $2(2)$ & - \\
\hline
\end{tabular}

Means (SDs) of response times, error rates and EEG amplitudes for congruent and incongruent trials of the Stroop task for both younger and older adults at both pre- and posttest

Analysis of RTs indicated effects of Congruency and Time. All participants responded more slowly on incongruent trials when compared with congruent trials (i.e., the Stroop effect; $b=0.22, S E=0.01, t=19.52, p<0.005$ ), with a significant Stroop effect for younger adults, and older adults, at pretest and posttest $(p<0.001$ for all four comparisons). A significant interaction between Age and Congruency $(b=0.13, S E=0.02, t=-5.64, p<0.005)$ 
indicated that the Stroop effect was stronger for older adults than younger adults

The RT data revealed a main effect of time $(b=-0.18$, $S E=0.01, t=-16.51, p<0.005)$, with participants performing faster overall post-exercise than pre-exercise. A significant Time $\times$ Age interaction was observed $(b=-0.08$, $S E=0.02, t=-3.69, p<0.005)$ as older adults' RTs decreased more following exercise (Fig. 3). Older adults had a significant reduction in $\mathrm{RT}$ from pre-exercise to post-exercise, $t(28)=7.73, p<0.001$, as did younger adults, $t(29)=6.87$, $p<0.001$, but older adults had a significantly greater decrease in RT from pre-exercise to post-exercise compared to younger adults $(p<0.001)$. There was no main effect of Exercise condition $(b=-0.05, S E=-0.06, t=-0.78, p=0.44)$ and no Exercise condition $\times$ Time interaction $(b=-0.02$, $S E=0.02, t=-0.83, p=0.41)$.

\section{Electrophysiological data}

Based on topographic maps of the cortex illustrating areas of peak activation across time, the $\mathrm{Cz}$ electrode was chosen as the site for ERP analysis. The time window of $320-700 \mathrm{~ms}$ post-stimulus presentation was selected based on the findings of previous studies [13, 17]. Figure 4 shows the grand average ERP waveforms for $\mathrm{Cz}$ for the congruent and incongruent trials for both younger and older adults.

Significant main effects of Age and Time were observed, with mean amplitudes lower for older adults than younger adults $(b=-2.73, S E=0.59, t=-4.64, p<0.005)$ and mean amplitude increased from pretest to posttest $(b=0.57$, $S E=0.28, t=2.06, p=0.04)$. There was an Age $\times$ Congruency interaction, where older adults exhibited greater ERP amplitude during incongruent Stroop trials compared with congruent trials than younger adults $(b=0.78$, $S E=0.32, t=2.47, p=0.01$ ). There was also a three-way interaction between BMI, Time and Congruency $(b=0.27$, $S E=0.11, t=2.47, p=0.01$, Fig. 5). At pre-exercise, there was a nonsignificant negative relationship between BMI and interference effect in younger adults $(r=-0.29$, $p=0.16$ ), and a nonsignificant positive relationship between BMI and interference effect in older adults $(r=0.26, p=0.21)$. At post-exercise, there was a
Fig. 3 a Younger and older adult response time performance and $\mathbf{b}$ Stroop interference effect (Incongruent-Congruent) in both Track and Game Conditions from pre- to postexercise. a A significant twoway Time $\times$ Age interaction $(b=-0.08, S E=0.02$, $t=-3.69, p<0.005)$ demonstrating that older adults showed a greater decrease in RT from pre- to posttest compared to younger adults across both conditions. b All participants responded more slowly to incongruent probes when compared with congruent probes $(b=0.22, S E=0.01$, $t=19.52, p<0.005)$. No significant changes on Stroop interference effect are observed from pre- to posttest
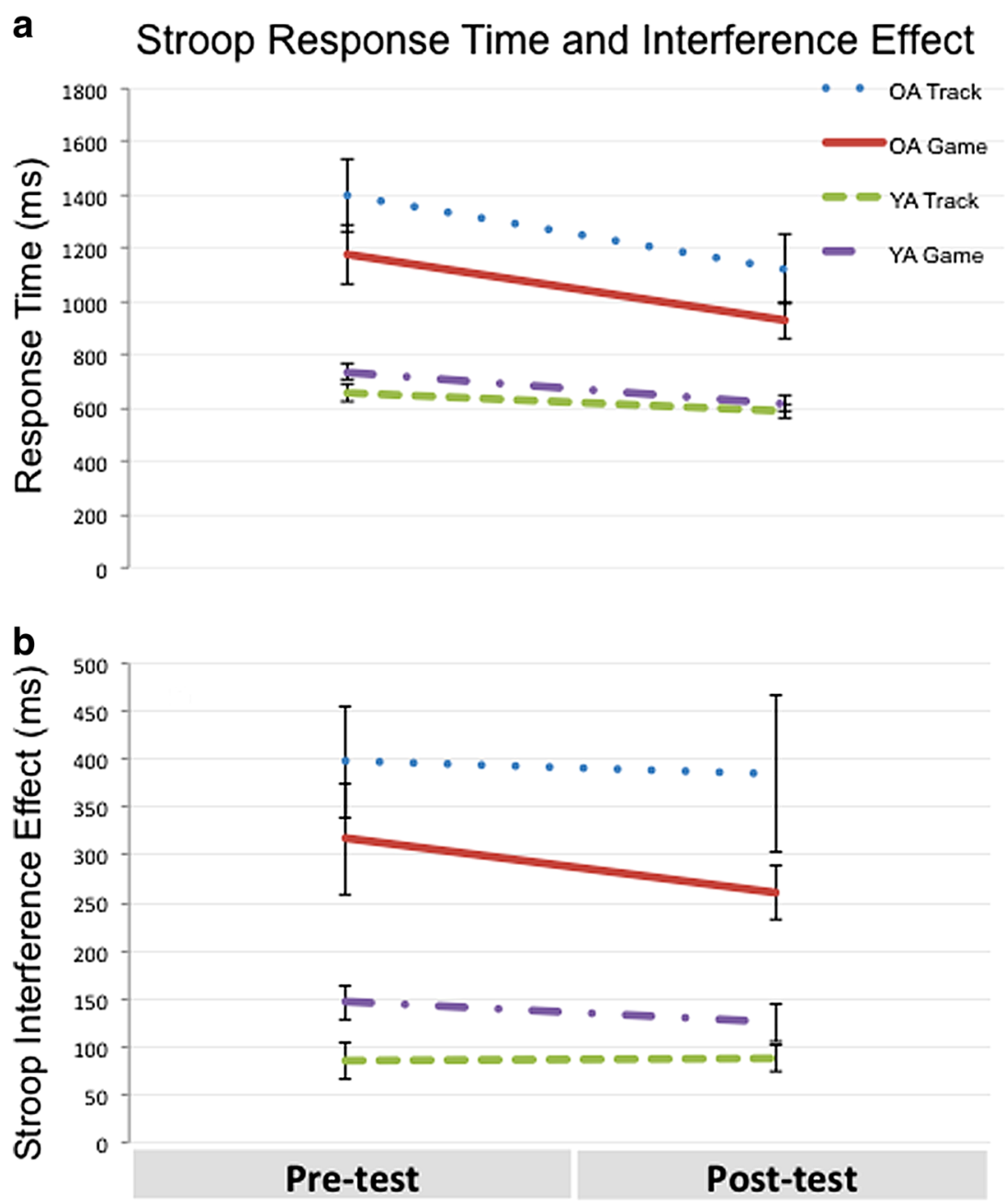
Fig. 4 Grand averages of mean peak amplitudes at the $\mathrm{Cz}$ electrode grand averaged ERP's at the $\mathrm{Cz}$ electrode site for younger adults and older adults at pre- and posttest. The $Y$ axis intersects the $X$ axis at time of stimulus presentation. Each tick mark represents $100 \mathrm{~ms}$

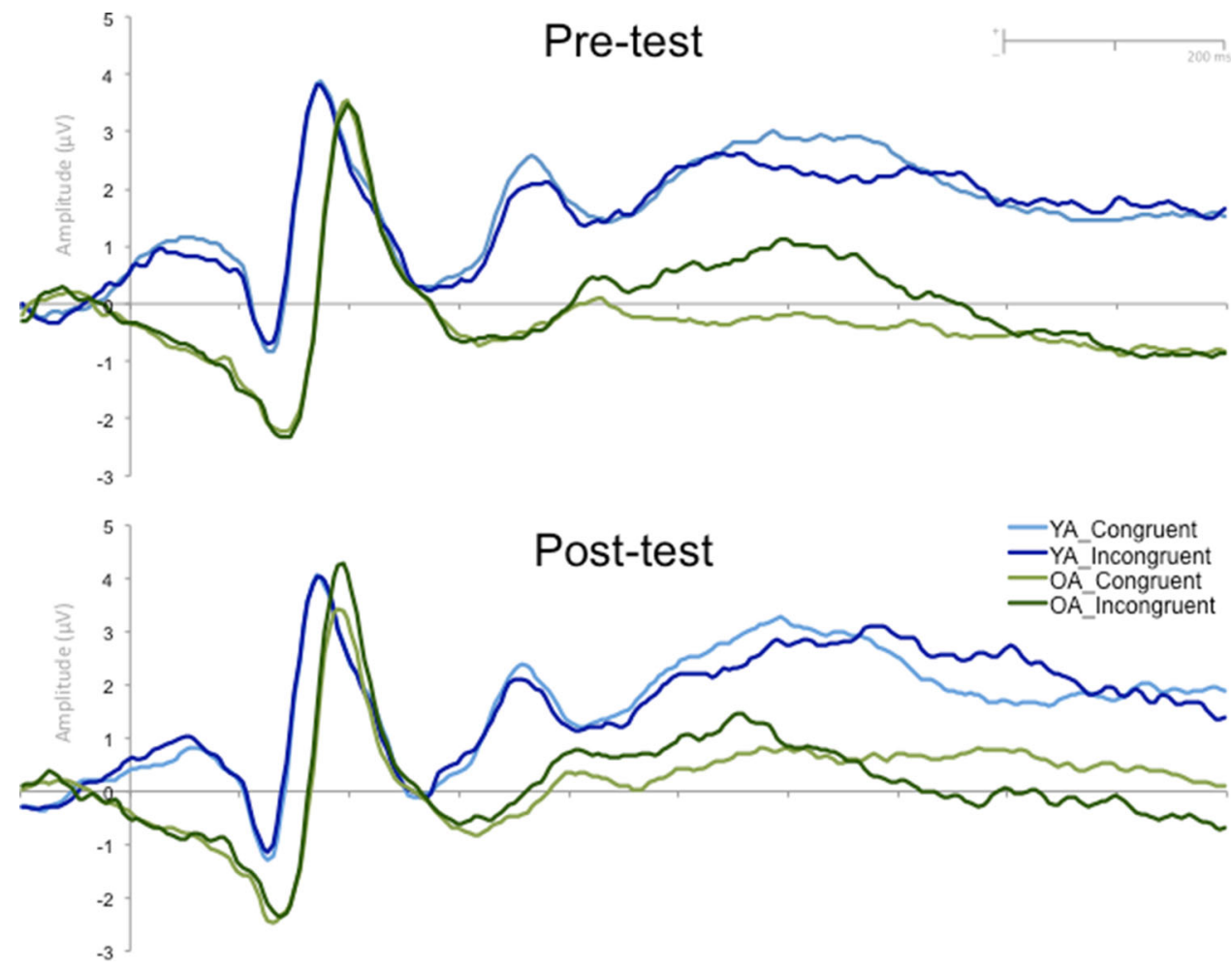

Relationship between BMI and Interference Effect by Time

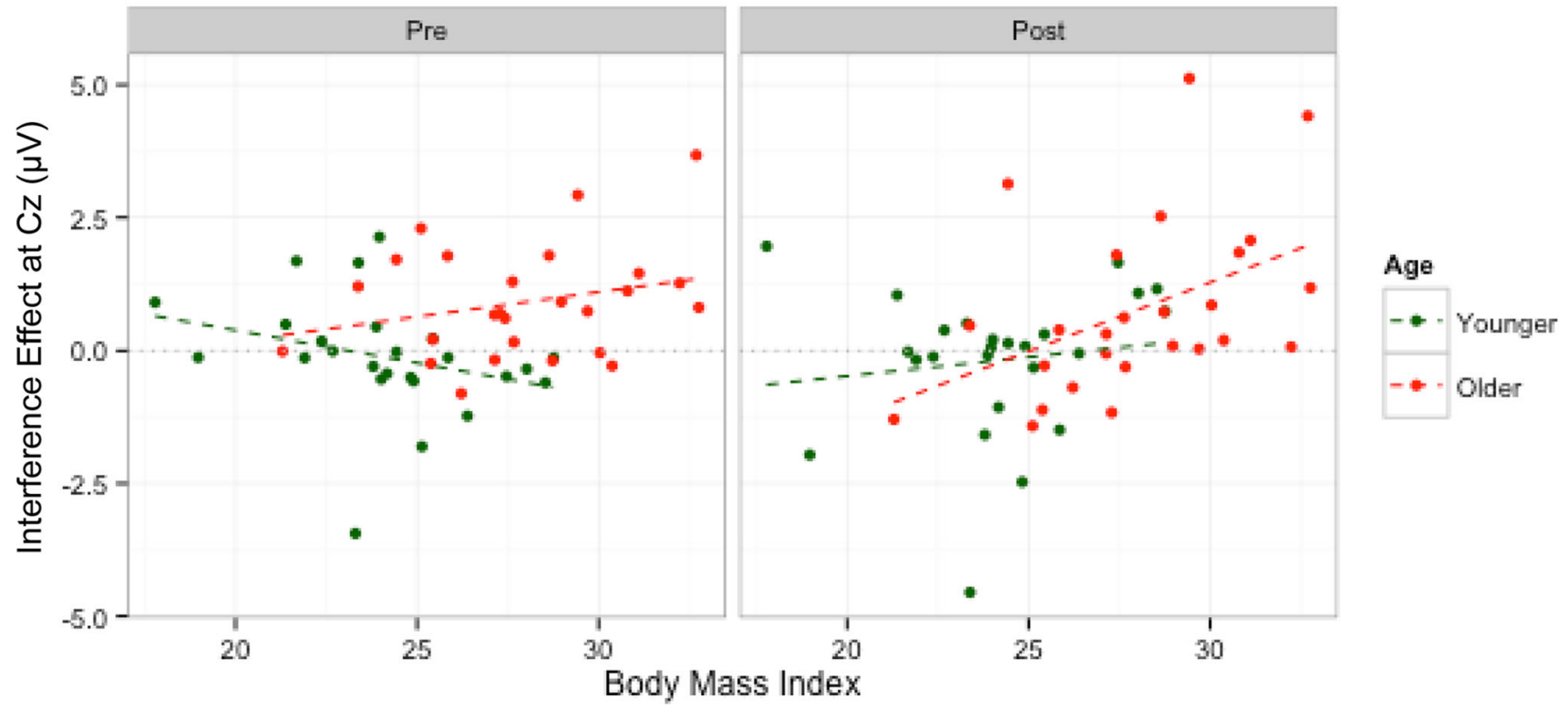

Fig. 5 Relationship between BMI and interference effect by Time for younger and older adults. There was a significant three-way interaction, BMI $\mathrm{x}$ Condition $\mathrm{x}$ Time $(b=0.27, S E=0.11$, $t=2.47, p=0.01$ ) where at pre-exercise there was a negative relationship between BMI and interference effect in younger adults

negligible positive relationship between BMI and interference effect in younger adults $(r=0.14, p=0.51)$, and the positive relationship between BMI and interference effect but a positive relationship between BMI and interference effect in older adults. Post-exercise, there was a negligible positive relationship between BMI and interference effect in younger adults, and the positive relationship between BMI and interference effect strengthened in older adults to a significant moderately strong relationship

strengthened in older adults to a significant moderately strong relationship $(r=0.45, p=0.02)$. The difference in relationships between pre-exercise and post-exercise was 
significant for the younger adults $(z=2.15, p=0.03)$, but not the older adults $(z=1.10, p=0.27)$. On average, the relationship between BMI and mean amplitude differed across age groups $(b=0.47, \quad S E=0.20, \quad t=2.35$, $p=0.02)$; a moderate positive correlation was observed for older adults $(r=0.38, p=0.06)$, and a nonsignificant negative correlation was observed for younger adults $(r=-0.18, p=0.39)$.

\section{Discussion}

The present study examined the effects of a single bout of standard aerobic exercise compared to interactive mental and aerobic exercise on the behavioral and electrophysiological functioning of younger and older adults. Previous intervention research found that, compared with a program of standard physical exercise, exergaming resulted in greater benefits on tests of executive function in older adults [10]. The current study focused on the acute effects of exercise on executive functioning and predicted that participants engaging in exergaming would receive an added benefit compared to those in the standard physical exercise condition. Although we found effects of exercise on outcomes, we found no significant differences between types of exercise performed (Fig. 6).

Notably, we found generalized benefits of exercise on behavioral performance, regardless of exercise condition. Participants were faster at posttest than pretest, and older adults experienced a greater overall decrease in RT when compared with younger adults, suggesting greater benefits of exercise on speed of processing in older adults when compared with younger adults. These findings are consistent with previous research demonstrating that an acute bout of exercise leads to enhanced cognitive performance, in particular when completing the Stroop task [18, 19].

The null effect of exercise condition may be attributed to several factors. One possibility is that the benefits of combined physical and cognitive activity interventions are only observed after an extended period of exergaming training and practice. Other factors may moderate effects in an acute bout condition including the nature of the game being played while exercising (e.g., simple versus complex; competitive versus cooperative), the cognitive load associated with the combined physical and cognitive activity, the perceived acceptability of the exergaming activity, among others. In one acute bout study comparing standard aerobic exercise to exergaming, O'Leary and colleagues [20] focused on younger adults and found no significant improvements in performance as a result of exergaming. The authors speculated that, under certain test conditions, where cognitive load is too high, effects afforded by traditional physical exercise might in fact be negated when one engages in simultaneous physical activity and virtual reality interactive gaming. Given that exergaming involves a set of novel and constantly changing stimuli, it is possible that the short-term effects of exergaming could be to increase cognitive load, which may make the performance on subsequent tasks more taxing. Further research is needed to examine these issues.

In the electrophysiological data, significant differences were observed between the mean amplitudes of incongruent and congruent trials among the older adults. Incongruent trials were greater in mean amplitude (i.e., more positive) than congruent trials. Furthermore, we observed a trend from pre-exercise to post-exercise whereby there was a decrease in the ERP difference between incongruent and congruent trials in older adults, suggesting a reduction in the interference effect.

More generally, we found that younger adults had significantly larger ERP amplitudes overall than older adults. Interestingly, we also found that post-exercise, there was an increase in overall amplitudes for both the older and younger adults, suggesting that exercise may have facilitated the recruitment of additional cognitive resources for the processing of both congruent and incongruent trials.

We also included BMI as a continuous predictor of the mean amplitudes in the ERP data analysis. In the current study, we found a moderate association between BMI values and participants' self-reported level of daily physical activity, indicating that higher BMI values are associated with lower levels of daily physical activity among all of our participants. We found an interesting three-way interaction between BMI, Time and Congruency, whereby the interference effect changed from pre-exercise to postexercise for both younger and older adults. Specifically, there was a significant positive relationship between BMI and the interference effect at post-exercise for older adults, which was not seen for younger adults or for older adults at pre-exercise. Thus, at post-exercise, older adults with a higher BMI exhibited a larger interference effect.

In the current study, older adults with a higher BMI may have exerted more physical energy and cognitive resources in their effort to complete the aerobic exercise session, with fewer resources available for performance on the Stroop task post-exercise, which in turn resulted in a larger ERP interference effect relative to peers with lower BMI. This indicates that, as opposed to standard exercise, the increased cognitive load of the exergaming condition may have made performance on subsequent tasks more taxing [21].

There are several limitations to the current study. It is possible that the concomitant cognitive task in the exergaming condition could have been inefficient in mobilizing the cognitive processes necessary to boost performance on the Stroop task in the context of an acute bout of exercise. Also, the sample size was relatively small which may have decreased the statistical power of the 


\section{BMI-Interference Effect by Age, Time and Condition}

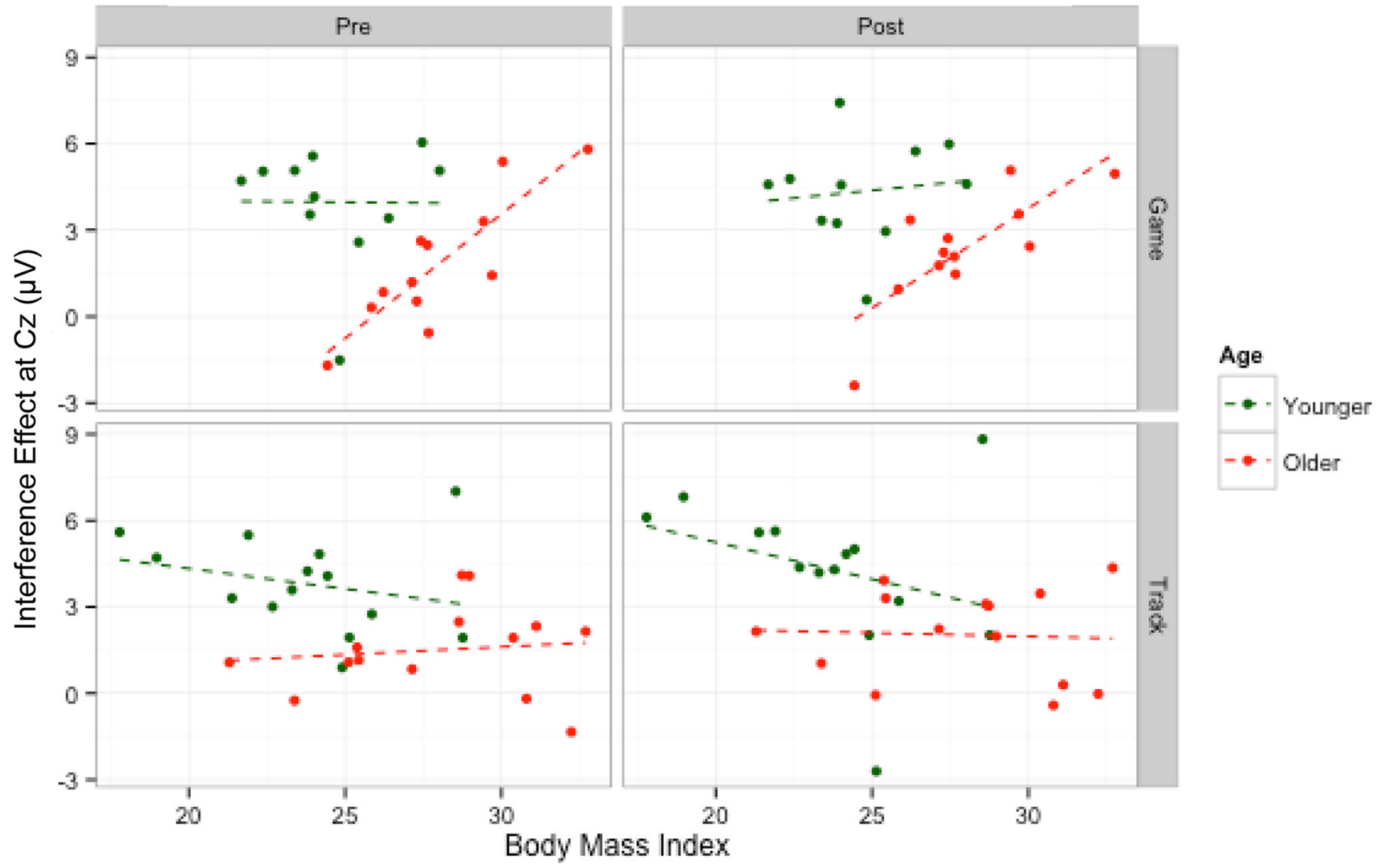

Fig. 6 BMI-interference effect (Incongruent-Congruent trials) by Time, Age and Condition. The difference in relationships between preexercise and post-exercise was significant for the younger adults $(z=2.15, p=0.03)$, but not the older adults $(z=1.10, p=0.27)$

analyses. Finally, incorporating a formal measure of fitness level of each participant would also have allowed us to more efficiently distinguish and attribute the observed effects to fitness level, exercise or a combination of both.

Overall, our findings support the hypothesis that an acute bout of exercise enhances cortical processing and cognitive performance as evidenced by the increased mean amplitudes of the ERPs and faster RT's at post-exercise in both younger and older adults. Furthermore, compared with younger adults, we observed a greater reduction in RT's for older adults at post-exercise, suggesting that older adults may have benefitted more from the acute exercise session than younger adults. We also found that older adults with lower BMI level demonstrated a smaller ERP interference effect post-exercise compared to their higher BMI counterparts. Taken together with findings from previous research showing no improvement in performance for a control (resting) group on the Stroop task [18] and studies demonstrating the moderating role of fitness level on electrophysiological task performance [5, 22], the current study lends additional support to the hypothesis that an acute bout of exercise can improve performance on cognitive tasks and these effects may be moderated by physical fitness levels.

In summary, the current study suggests that an acute bout of exergaming as administered in this context does not facilitate enhanced cognitive performance when compared to standard aerobic exercise. However, future research should seek to determine the differential neural mechanisms that contribute to improved cognitive performance after a long-term intervention when compared to an acute session and whether different types of exergaming may be more effective than others at producing improvements in cognitive performance in an acute bout context. This would allow us to tailor exercise interventions for maximum efficiency and to promote increased compliance among exercisers.

\section{Compliance with ethical standards}

Conflict of interest On behalf of all authors, the corresponding author states that there is no conflict of interest.

Statement on Human Rights All procedures performed in studies involving human participants were in accordance with the ethical standards of the institutional and/or national research committee and 
with the 1964 Helsinki declaration and its later amendments or comparable ethical standards.

Informed Consent Informed consent was obtained from all individual participants included in the study.

\section{References}

1. Bishop NA, Lu T, Yankner BA (2010) Neural mechanisms of ageing and cognitive decline. Nature 464:529-535

2. Erickson KI, Voss MW, Prakash RS et al (2011) Exercise training increases size of hippocampus and improves memory. Proc Natl Acad Sci U S A 108:3017-3022

3. Kramer AF, Erickson KI, Colcombe SJ (2006) Exercise, cognition, and the aging brain. J Appl Physiol 101:1237-1242

4. Colcombe S, Kramer AF (2003) Fitness effects on the cognitive function of older adults a meta-analytic study. Psychol Sci $14: 125-130$

5. Stroth S, Kubesch S, Dieterle K et al (2009) Physical fitness, but not acute exercise modulates event-related potential indices for executive control in healthy adolescents. Brain Res 1269:114-124

6. Chang YK, Labban J, Gapin J et al (2012) The effects of acute exercise on cognitive performance: a meta-analysis. Brain Res 1453:87-101

7. Puccioni O, Vallesi A (2012) Conflict resolution and adaptation in normal aging: the role of verbal intelligence and cognitive reserve. Psychol Aging 27:1018

8. West R, Alain C (2000) Age-related decline in inhibitory control contributes to the increased Stroop effect observed in older adults. Psychophysiology 37:179-189

9. West R, Moore K (2005) Adjustments of cognitive control in younger and older adults. Cortex 41:570-581

10. Anderson-Hanley C, Arciero PJ, Brickman AM et al (2012) Exergaming and older adult cognition: a cluster randomized clinical trial. Am J Prev Med 42:109-119

11. Maillot P, Perlot A, Hartley A (2012) Effects of interactive physical-activity video-game training on physical and cognitive function in older adults. Psychol Aging 27:589-600
12. Badzakova-Trajkov G, Barnett KJ, Waldie KE et al (2009) An ERP investigation of the Stroop task: the role of the cingulate in attentional allocation and conflict resolution. Brain Res 1253:139-148

13. Liotti M, Woldorff MG, Perez R et al (2000) An ERP study of the temporal course of the Stroop color-word interference effect. Neuropsychologia 38:701-711

14. Hogan M, Kiefer M, Kubesch S et al (2013) The interactive effects of physical fitness and acute aerobic exercise on electrophysiological coherence and cognitive performance in adolescents. Exp Brain Res 229:85-96

15. Chodzko-Zajko WJ, Proctor DN, Fiatarone Singh MA et al (2009) American College of Sports Medicine Position stand. Exercise and physical activity for older adults. Med Sci Sports Exerc 41:1510-1530. doi:10.1249/MSS.0b013e3181a0c95c

16. Kerns JG, Cohen JD, MacDonald AW et al (2004) Anterior cingulate conflict monitoring and adjustments in control. Science 303:1023-1026

17. Gratton G, Coles MG, Donchin E (1983) A new method for offline removal of ocular artifact. Electroencephalogr Clin Neurophysiol 55:468-484

18. Hyodo K, Dan I, Suwabe K et al (2012) Acute moderate exercise enhances compensatory brain activation in older adults. Neurobiol Aging 33:2621-2632

19. Yanagisawa H, Dan I, Tsuzuki D et al (2010) Acute moderate exercise elicits increased dorsolateral prefrontal activation and improves cognitive performance with Stroop test. Neuroimage 50:1702-1710

20. O'Leary KC, Pontifex MB, Scudder MR et al (2011) The effects of single bouts of aerobic exercise, exergaming, and videogame play on cognitive control. Clin Neurophysiol 122:1518-1525

21. Robert G, Hockey J (1997) Compensatory control in the regulation of human performance under stress and high workload: a cognitive-energetical framework. Biol Psychol 45:73-93

22. Tsai CL, Chen FC, Pan CY et al (2014) Impact of acute aerobic exercise and cardiorespiratory fitness on visuospatial attention performance and serum BDNF levels. Psychoneuroendocrinology 41:121-131 